\title{
SLIT2/ROBO2 signaling pathway inhibits nonmuscle myosin IIA activity and destabilizes kidney podocyte adhesion
}

\author{
Xueping Fan, ${ }^{1}$ Hongying Yang, ${ }^{2}$ Sudhir Kumar, ${ }^{1}$ Kathleen E. Tumelty, ${ }^{2}$ Anna Pisarek-Horowitz, \\ Hila Milo Rasouly, ${ }^{1}$ Richa Sharma, ${ }^{1}$ Stefanie Chan, ${ }^{1}$ Edyta Tyminski, ${ }^{2}$ Michael Shamashkin, ${ }^{2}$ \\ Mostafa Belghasem, ${ }^{3}$ Joel M. Henderson, ${ }^{3}$ Anthony J. Coyle, ${ }^{2}$ David J. Salant, ${ }^{1}$ Stephen P. Berasi, ${ }^{2}$ \\ and Weining Lu ${ }^{1}$ \\ ${ }^{1}$ Renal Section, Department of Medicine, Boston University Medical Center, Boston, Massachusetts, USA. ${ }^{2}$ Centers for \\ Therapeutic Innovation, Pfizer Inc., Boston, Massachusetts, USA, ${ }^{3}$ Department of Pathology and Laboratory Medicine, \\ Boston University Medical Center, Boston, Massachusetts, USA.
}

\begin{abstract}
The repulsive guidance cue SLIT2 and its receptor ROBO2 are required for kidney development and podocyte foot process structure, but the SLIT2/ROBO2 signaling mechanism regulating podocyte function is not known. Here we report that a potentially novel signaling pathway consisting of SLIT/ ROBO Rho GTPase activating protein 1 (SRGAP1) and nonmuscle myosin IIA (NMIIA) regulates podocyte adhesion downstream of ROBO2. We found that the myosin II regulatory light chain (MRLC), a subunit of NMIIA, interacts directly with SRGAP1 and forms a complex with ROBO2/ SRGAP1/NMIIA in the presence of SLIT2. Immunostaining demonstrated that SRGAP1 is a podocyte protein and is colocalized with ROBO2 on the basal surface of podocytes. In addition, SLIT2 stimulation inhibits NMIIA activity, decreases focal adhesion formation, and reduces podocyte attachment to collagen. In vivo studies further showed that podocyte-specific knockout of Robo2 protects mice from hypertension-induced podocyte detachment and albuminuria and also partially rescues the podocyte-loss phenotype in Myh9 knockout mice. Thus, we have identified SLIT2/ ROBO2/SRGAP1/NMIIA as a potentially novel signaling pathway in kidney podocytes, which may play a role in regulating podocyte adhesion and attachment. Our findings also suggest that SLIT2/ ROBO2 signaling might be a therapeutic target for kidney diseases associated with podocyte detachment and loss.
\end{abstract}

Conflict of interest: This project was supported, in part, by a research grant from the Pfizer Centers for Therapeutic Innovation.

Submitted: February 5, 2016 Accepted: October 6, 2016 Published: November 17, 2016

Reference information: JCI Insight. 2016;1(19):e86934. doi:10.1172/jci.insight.86934.

\section{Introduction}

The kidney glomerular filtration barrier, composed of the fenestrated capillary endothelial cells, the glomerular basement membrane (GBM), and podocytes, restricts the permeability of blood cells and plasma proteins. Podocytes are specialized epithelial cells that cover the outer surface of the GBM. The actin-rich interdigitating foot processes from neighboring podocytes create filtration slits bridged by a semiporous slit-diaphragm that forms the final barrier to protein permeation (1). The interconnecting podocytes also adhere to the GBM, a dense network structure of secreted extracellular matrix molecules including type IV collagen and laminin, through cell-matrix adhesion receptors such as integrins and dystroglycans (2, 3 ). The sophisticated function of glomerular filtration relies on normal podocyte adhesion at both the slitdiaphragm and the interface with the GBM, as genetic mutations of slit-diaphragm proteins such as nephrin and podocyte-GBM adhesion proteins such as integrin $\alpha 3$ are associated with hereditary forms of nephrotic syndrome $(1,4)$. Podocytes coordinate signals from cell junctions and cell-matrix interactions in response to environmental cues and thereby regulate filtration (2). Recent studies have shown that the slit-diaphragm protein nephrin regulates podocyte structure and function by promoting actin polymerization $(5,6)$, whereas the SLIT2/ROBO2 signaling pathway inhibits nephrin-associated actin polymerization (7).

Roundabout (ROBO) family proteins, ROBO1-4, are single-pass transmembrane receptors for SLIT1-3. Secreted SLITs were originally found as repulsive guidance cues for axon pathfinding and migrating neurons during the development of the nervous system (8). The extracellular region of ROBO $1 / 2$ contains 5 Ig domains and 3 fibronectin type III (FNIII) repeats $(9,10)$. While both Ig domains 1 and 2 interact with 
SLIT (11), Ig domain 1 is the primary binding site (12). The intracellular domain of ROBO1/2 contains 4 conserved cytoplasmic (CC) sequences named CC0, CC1, CC2, and CC3 (9, 10, 13). CC0 and CC1 contain multiple tyrosines, while CC2 and CC3 are proline rich. Upon SLIT binding, ROBO1/2 receptors interact with GTPase-activating proteins (SRGAPs) to downregulate small GTPase Cdc42 activity to inhibit F-actin polymerization (14). Signaling components such as Ena/Abl, Dock, PAK, Sos, Cables, and $\mathrm{Ca}^{2+}$ have been shown to be involved in SLIT/ROBO repulsive signaling pathways (15-18).

SLIT2/ROBO2 signaling also plays a crucial role during early kidney development and ureteric bud outgrowth. Mouse mutants that lack Slit2 or Robo2 develop supernumerary ureteric buds, which lead to a broad spectrum of urinary tract phenotypes including duplex kidneys, abnormal ureterovesical junctions, and hydronephrosis $(19,20)$. Disruption of $\mathrm{ROBO} 2$ in humans causes congenital anomalies of the kidneys and urinary tracts (CAKUT), and point mutations of ROBO2, SLIT2, and SRGAP1 have also been identified in patients with CAKUT and vesicoureteral reflux (20-23). We have recently found that ROBO2 is expressed by podocytes and forms a protein complex with nephrin through the NCK adaptor protein to inhibit nephrin-induced actin polymerization, and loss of Robo2 partially rescues the podocyte foot process structure defects in nephrin-null mice (7). However, the complete mechanism underlying the renoprotective effect of ROBO2 signaling loss in the podocyte remains unclear.

Nonmuscle myosin II (NMII) is a bipolar, contractile protein composed of 2 nonmuscle myosin heavy chains (NMHCs), 2 myosin regulatory light chains (MRLCs), and 2 myosin essential light chains (MELCs) $(24,25)$. There are 3 nonmuscle NMHC isoforms, IIA, IIB, and IIC, which are encoded by the MYH9, MYH10, and MYH14 genes, respectively (26). Each NMHC contains an N-terminal globular motor domain that moves F-actin as it hydrolyzes ATP and a C-terminal tail that binds to another NMHC pair C-tail to form a bipolar filament $(24,26,27)$. NMII has diverse functions in cell contractility, cytokinesis, locomotion, and adhesion, and its activity is primarily controlled by MRLC phosphorylation, which is regulated by kinases and phosphatases (26). Mutations in the human MYH9 gene cause rare MYH9related diseases characterized by autosomal dominant glomerulonephritis, proteinuria, and podocyte foot process effacement together with blood disorders such as thrombocytopenia and giant platelets (28). Loss of $M y h 9$ in the mouse metanephric mesenchyme results in failure to maintain renal tubules during kidney development, and podocyte-specific deletion of $M y h 9$ predisposes adult mice to induced experimental glomerulopathy (29-31). However, the mechanisms responsible for the latter have not been defined. Although monogenic MYH9-related glomerular diseases are rare, together with the mouse studies, they identify a potential pathway that may be relevant to more common forms of proteinuric nephropathies.

We herein report a potentially novel ROBO2/SRGAP1/NMIIA signaling pathway in kidney podocytes. Through combined in vitro and in vivo studies, we have found that ROBO2/SRGAP1/NMIIA form a complex in podocytes. We found that increased SLIT2/ROBO2 signaling decreased phospho-MRLC (p-MRLC) levels and inhibited NMII activity in podocytes, leading to reduced formation of focal adhesion and decreased podocyte adhesion to collagen-coated plates. Mouse genetic studies further demonstrated that podocyte-specific knockout of Robo2 protected mice from hypertension-induced podocyte detachment and albuminuria and partially rescued the podocyte loss and albuminuria phenotype in the Myh 9 knockout mice. These results suggest that SLIT2/ROBO2 signaling inhibits NMII and reduces podocyte adhesion in kidney glomeruli.

\section{Results}

ROBO2 interacts with SRGAP1 and forms a complex with NMIIA. SRGAP proteins, important components of the SLIT/ROBO signaling pathway during neuronal development, have an F-BAR domain in the N-terminus, a GAP domain in the middle, and an SH3 domain near the C-terminus (Figure 1A) (14). In neurons, ROBO1, upon SLIT stimulation, binds to the SH3 domain of SRGAP1 via its CC3 proline-rich motif to inhibit actin polymerization (14). To determine if ROBO2 also binds to SRGAP1, we specifically tested the interaction between the intracellular domain (ICD) of ROBO2 and the SH3 domain of SRGAP1 using a yeast 2-hybrid assay and observed a positive interaction (Figure 1B). Like ROBO1, domain mapping in ROBO2 showed that the CC3 motif in ROBO2 is responsible for the interaction with the SH3 domain of SRGAP1.

Because a recent study linked SRGPA3 (a homolog of SRGAP1) to MRLC in mammary epithelial cells (32), we hypothesized that SRGAP1 is also associated with MRLC, the 172-residue subunit of NMII. To test this hypothesis, we examined whether the F-BAR domain of SRGAP1 interacts directly with MRLC in a yeast 2-hybrid assay. A positive interaction between the F-BAR domain of SRGAP1 


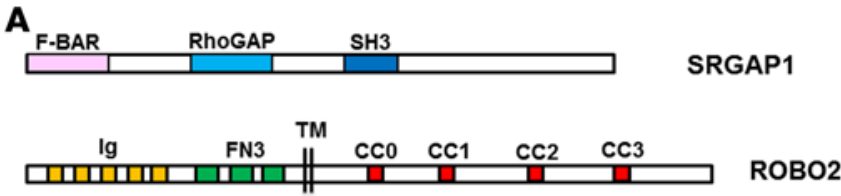

B

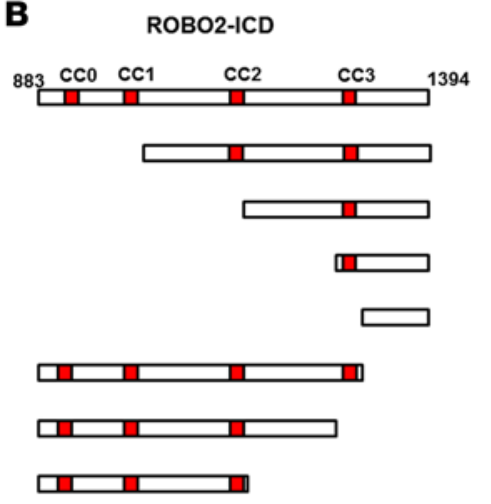

SRGAP1

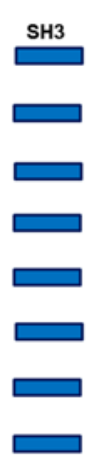

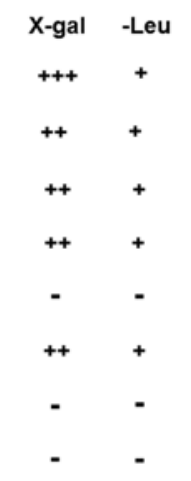

C
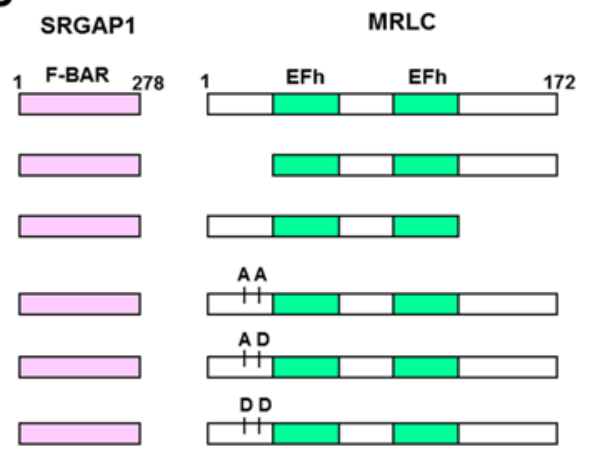

X-gal -Leu
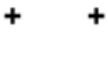

D

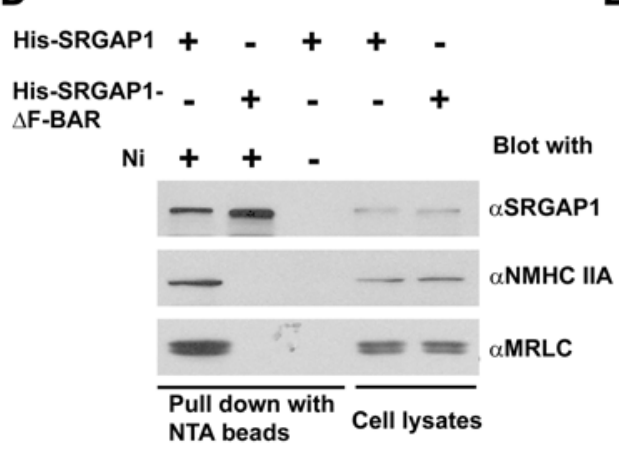

$\mathbf{F}$

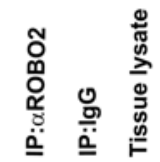

$\alpha$ ROBO2

$\alpha$ SRGAP1

$\alpha$ MRLC

$\alpha \mathrm{p}-M R L C$

$\alpha$ NMHC ॥A

Figure 1. ROBO2, SRGAP1, and nonmuscle myosin IIA (NMIIA) form a complex. (A) Diagrams showing the domain structure of SRGAP1 and ROBO2 proteins. Abbreviations: F-BAR, FES-CIP4 homology and Bin/amphiphysin/Rvs domain; RhoGAP, GTPase-activator protein (CAP) for Rho-like GTPases domain; SH3, Src homolog 3 domain; Ig, immunoglobulin domain; FN3, fibronectin type 3 domain; CCO, CC1, CC2, CC3, cytoplasmic conserved region 0, 1, 2, and 3; TM, transmembrane domain. (B) Yeast 2-hybrid assay shows a positive interaction between the ROBO2 intracellular domain (ROBO2-ICD) and the SH3 domain of SRGAP1. The interaction is mediated by the proline-rich CC3 motif in ROBO2 and the SH3 domain in SRGAP1. LacZ reporter (X-gal): +++, yeast turned dark blue; ++, light blue; -, white in 24 hours. Leucine reporter (-Leu): +, yeast grew; -, yeast did not grow. Numbers indicate residue positions in the full-length ROBO2 protein. (C) Yeast 2-hybrid assay shows a positive interaction between the F-BAR domain of SRGAP1 and the myosin regulatory light chain (MRLC). Deletion of either N-terminal residues 1-33 or C-terminal amino acids 130-172 in MRLC abolishes the interaction. Substitutions of phosphorylation sites Thr18 and Ser19 with Ala-Ala, Ala-Asp, or Asp-Asp do not affect the interaction of MRLC with SRCAP1. EFh, EF-hand calciumbinding motif. Numbers indicate residue positions in the full-length proteins. (D) Full-length SRGAP1, not SRGAP1 with F-BAR deleted, coprecipitates with NMIIA heavy chain (NMHC IIA). His-myc-SRGAP1 or His-myc-SRGAP1- $\triangle$ F-BAR was expressed in HEK cells and precipitated with Ni-NTA beads (lanes 1 and 2) or control beads (lane 3) in the presence of SLIT2. (E) Full-length ROBO2, not ROBO2 without CC3 (ROBO2- $\triangle C C 3$ ), coprecipitates with SRCAP1, NMHC IIA, and MRLC. His-myc-ROBO2 or His-myc-ROBO2- $\triangle$ CC3 was expressed in HEK cells and precipitated with Ni-NTA beads (lanes 1 and 2) or control beads (lane 3) in the presence of SLIT2. (F) Coimmunoprecipitation of newborn mouse kidney endogenous ROBO2, SRGAP1, MRLC, p-MRLC, and NMHC IIA. Precipitates were prepared using mouse monoclonal anti-ROBO2 antibody and IgG antibody was used as a negative control.

and full-length MRLC was observed (Figure 1C). Deletion of either the N- or C-terminus of MRLC abolished the interaction, suggesting that full-length MRLC is required for the interaction with SRGAP1. Since MRLC phosphorylation at residues 18 (threonine) and 19 (serine) is crucial to activate NMII (26), we created mutant forms of MRLC in which the 2 phosphorylation sites were replaced by 2 alanines (AA), alanine and aspartic acid (AD), or 2 aspartic acids (DD) to mimic dephosphorylated, monophosphorylated, and diphosphorylated states, respectively. All modified MRLCs still interact with the F-BAR domain of SRGAP1, similar to the wild-type MRLC, suggesting that MRLC interaction with SRGAP1 is independent of MRLC phosphorylation at these 2 sites (Figure 1C). 

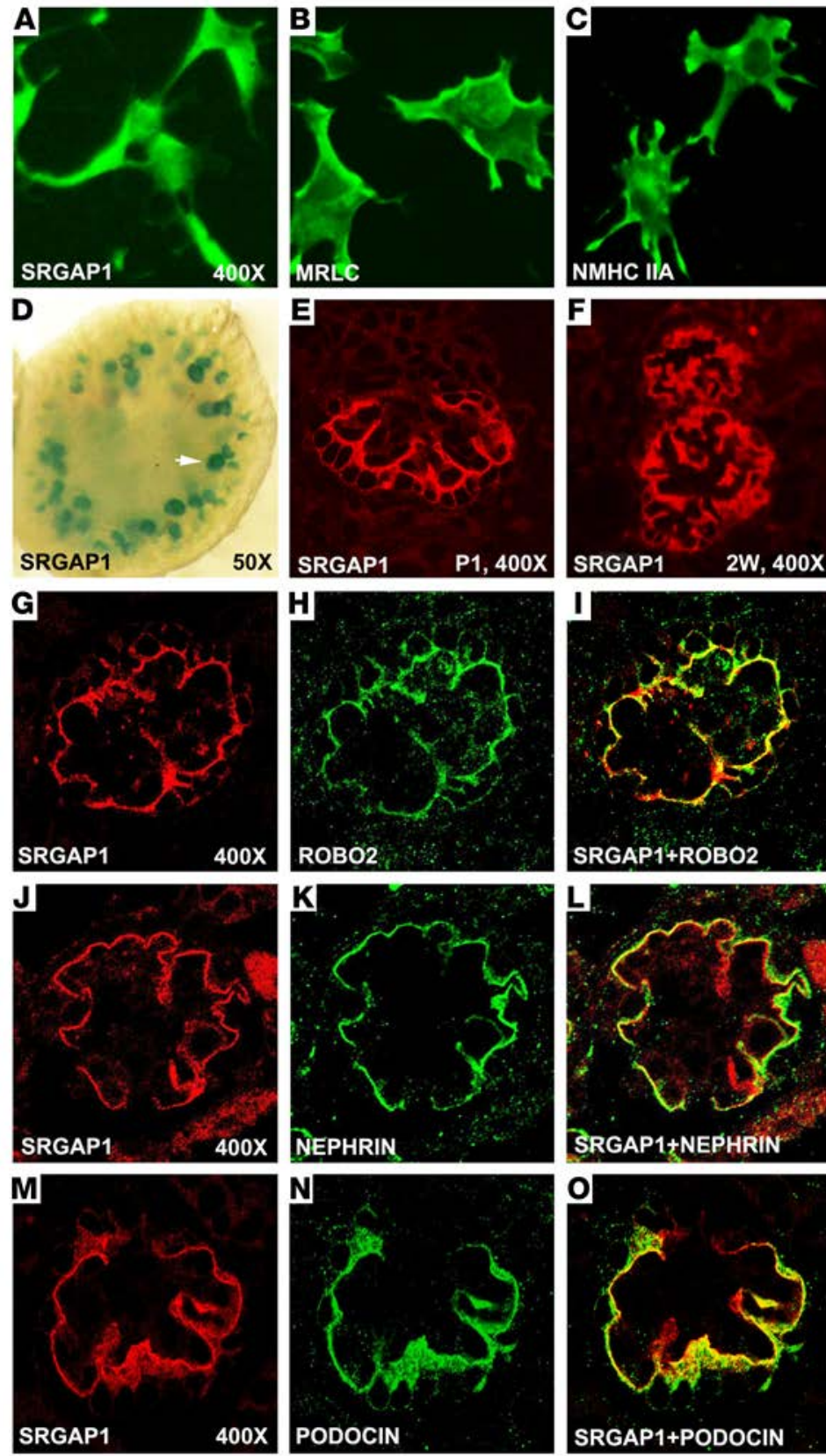
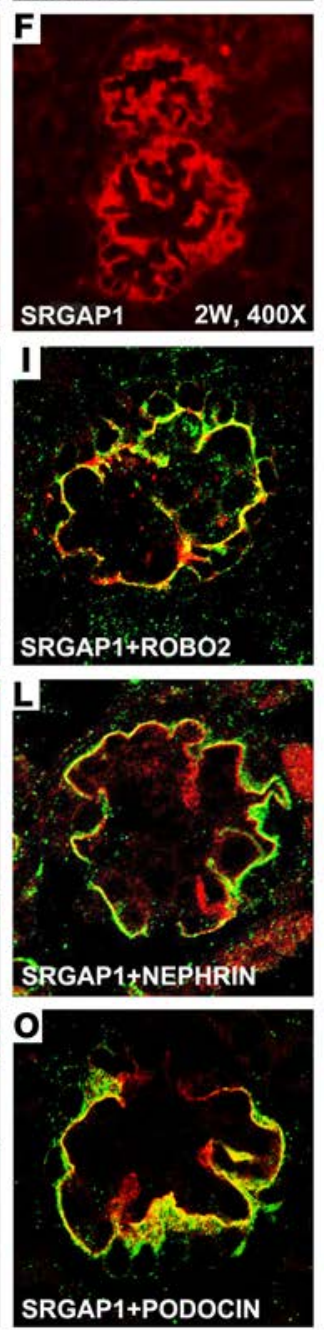

Figure 2. ROBO2, SRGAP1, and nonmuscle myosin IIA (NMIIA) are expressed in mouse podocytes. (A-C) Differentiated mouse podocytes express SRGAP1, myosin regulatory light chain (MRLC), and NMIIA heavy chain (NMHC IIA). $\times 400$ magnification. (D) LacZ staining shows that SRGAP1 is expressed in embryonic day 16.5 mouse glomeruli (white arrow) of a Srgap1-LacZ reporter mouse kidney. $\times 50$ magnification. ( $\mathbf{E}$ and $\mathbf{F}$ ) SRGAP1 protein is detected by immunofluorescence staining in mouse kidney glomeruli at postnatal day $1(\mathrm{P} 1)$ and 2 weeks of age $(2 \mathrm{~W}) . \times 400$ magnification (G-I) SRGAP1 (red) is colocalized with ROBO2 (green) in the $\mathrm{P} 1$ mouse glomeruli as detected by coimmunolabeling. $\times 400$ magnification. (J-L) SRGAP1 (red) is colocalized with nephrin (green) in the P1 mouse glomeruli as detected by coimmunolabeling. $\times 400$ magnification. (M-0) SRGAP1 (red) is colocalized with podocin (green) in the $\mathrm{P} 1$ mouse glomeruli as detected by coimmunolabeling. $\times 400$ magnification.

To further confirm the interaction between SRGAP1 and NMIIA, we performed protein pull-down assays. Histagged human SRGAP1 (His-SRGAP1) or SRGAP1 with the F-BAR domain deleted (His-SRGAP1- $\triangle$ F-BAR) was expressed in HEK cells and precipitated by Ni-NTA Histag-binding beads (Figure 1D). His-SRGAP1 precipitates, but not His-SRGAP1- $\triangle$ F-BAR precipitates, contain MRLC and NMHC IIA. To determine whether ROBO2, SRGAP1, and NMIIA form a complex, His-myc-ROBO2 or Hismyc-ROBO2- $\triangle \mathrm{CC} 3$ (binding site for SRGAP1 deleted) was expressed in HEK cells and pulled down by Ni-NTA beads. His-myc-ROBO2, but not His-myc-ROBO2- $\triangle \mathrm{CC} 3$, coprecipitates with SRGAP1, MRLC, and NMHC IIA (Figure 1E), indicating that ROBO2, SRGAP1, and NMIIA form a complex. This complex formation was also observed in vivo as the coimmunoprecipitates of $\mathrm{ROBO} 2$ from newborn mouse kidney lysates contain SRGAP1 and NMIIA, including MRLC, phosphorylated MRLC, and NMHC IIA (MYH9) (Figure $1 \mathrm{~F}$ ). This triple interaction was also confirmed in a podocyte cell line using the coprecipitation assay (Supplemental Figure 1; supplemental material available online with this article; doi:10.1172/jci.insight.86934DS1).

ROBO2, SRGAP1, and NMIIA are expressed in podocytes that also secrete functional SLIT2. We have previously reported that $\mathrm{ROBO} 2$ is expressed in kidney podocytes and is predominantly localized to the basal surface to regulate the F-actin cytoskeleton (7). NMIIA has also been reported to be expressed in podocytes (30). Since ROBO2, SRGAP1, and NMIIA are able to form a complex, we wanted to examine if these proteins, especially SRGAP1, are also expressed in podocytes and are colocalized with podocyte-specific markers. Using immunocytochemistry assays, we first detected expression of SRGAP1, MRLC, and NMHC IIA in differentiated mouse immortalized podocytes (33) (Figure 2, A-C and Supplemental Figure 2). To characterize the specificity of SRGAP1 expression in vivo, we took advantage of a Srgap1-LacZ reporter mouse line from the knockout mouse project (KOMP), in which the LacZ reporter is driven by the SRGAP1 promoter. We found that SRGAP1 transcript (marked by LacZ-positive signal) is specifically expressed in the glomeruli (Figure 2D). Immunofluorescence studies further confirmed the glomerulus-specific expression of SRGAP1 protein, and this expression pattern is maintained in postnatal mouse glomeruli (Figure 2, $\mathrm{E}$ and $\mathrm{F}$ ). Consistent with the ROBO2 expression pattern in podocytes (7), SRGAP1 is also localized to the basal surface of the podocyte and is colocalized with ROBO2 (Figure 2, G-I), nephrin (Figure 2, J-L), and podocin (Figure 2, M-O), 3 podocyte-specific markers.

We have previously reported that SLIT2 is expressed in adult mouse glomeruli and is coexpressed with 


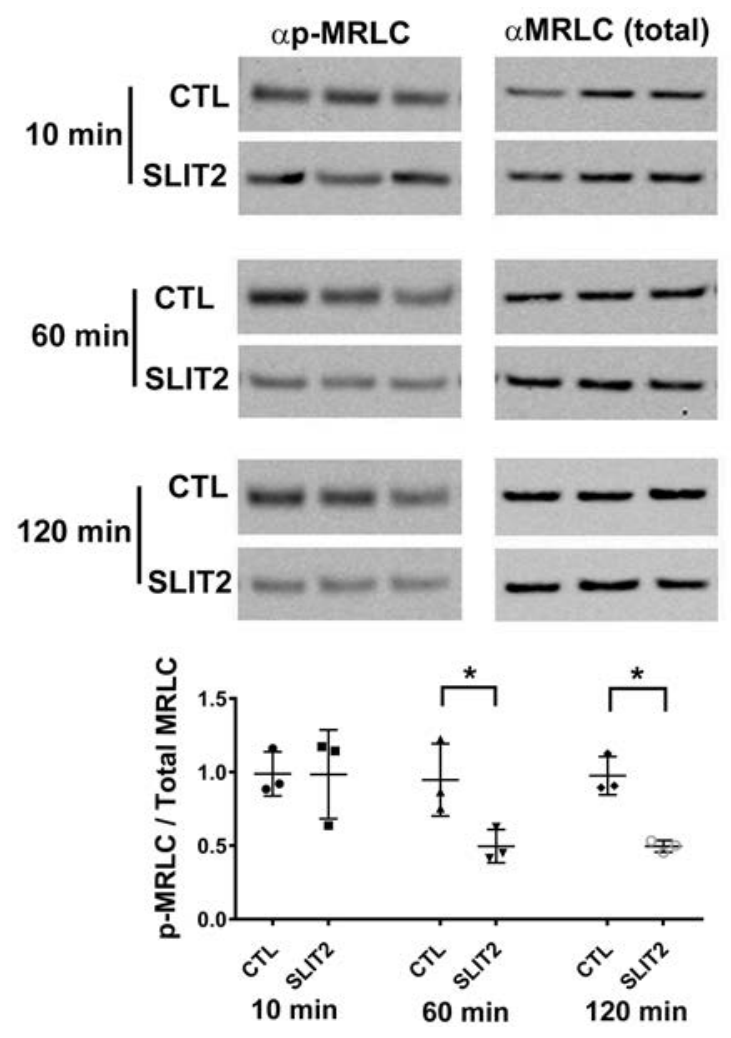

Figure 3. SLIT2 stimulation leads to a reduction of phosphorylated myosin regulatory light chain ( $p-M R L C)$ in differentiated podocytes. Western blot assay reveals decreased $p$-MRLC levels in differentiated mouse podocytes after SLIT2 treatment. Cells were stimulated by media containing recombinant SLIT2 $\mathrm{N}$-terminus or buffer for 10,60 , or 120 minutes before lysis. p-MRLC and total MRLC were detected by respective antibodies. Intensities of bands were measured and the ratio of $\mathrm{p}-\mathrm{MRLC} /$ total MRLC was calculated. Data are presented as the mean $\pm \mathrm{SEM}, n=3$. ${ }^{*} P<0.05$ compared with its control by 2 -tailed Student's $t$ test.

the podocyte marker synaptopodin (7). To determine if podocyte cell lines also secrete functional SLIT2, we performed in vitro neuronal migration coculture assays in which podocyte aggregates were cultured in proximity to rat brain tissue explants that were isolated from the anterior subventricular zone (SVZa) (20). Similar to the positive-control HEK cells that are transfected with recombinant SLIT2, both differentiated and nondifferentiated podocytes express SLIT2 (Supplemental Figure 3A) and repel SVZa neuron outgrowth (Supplemental Figure 3B), suggesting that these cultured podocytes indeed secrete functional SLIT2 that can bind and signal through ROBO2 receptors in SVZa neurons to inhibit cell migration.

SLIT2 stimulation decreases phosphorylation of MRLC and thereby inhibits NMII activity. Phosphorylation of MRLC is a crucial step to activate NMII (26). Since ROBO2 forms a complex with SRGAP1, MRLC, and NMHC IIA, we wanted to determine if SLIT2/ROBO2 signaling has an impact on the phosphorylation of MRLC to thereby affect NMII activity. We treated differentiated mouse podocytes with SLIT2 or control buffer for 10, 60, and 120 minutes and then examined p-MRLC and total MRLC levels by Western blotting using specific antibodies. We found that p-MRLC levels are significantly decreased at 60 or 120 minutes in the presence of SLIT2, whereas the level of the total MRLC is not affected (Figure 3). This finding of reduced MRLC phosphorylation by SLIT2 stimulation suggests that SLIT2/ROBO2 signaling inhibits NMII activity in podocytes.

SLIT2/ROBO2 signaling reduces differentiated mouse podocyte adhesion to collagen and decreases the formation of focal adhesions. In the nervous system, SLIT and ROBO inhibit neuronal migration and growth cone motility (34), but their effect on cell adhesion has not been explored. Since NMII is central in the control of cell adhesion (26) and our data indicate that NMII forms a complex with ROBO2 and SLIT2/ROBO2 signaling inhibits NMII activity in podocytes, we hypothesized that increased SLIT2/ROBO2 signaling would modulate podocyte adhesion. To examine this possibility, we generated 2 mouse podocyte cell lines through viral transduction: a ROBO2 podocyte cell line that overexpresses YFP-tagged ROBO2 and a vector control podocyte cell line. We first checked the expression level of ROBO2 in these 2 cell lines by Western blotting and found a high level of ROBO2 expression in the ROBO2-overexpressing podocytes as compared with the vector control cells (Figure 4A). To examine the effect of increased SLIT2/ROBO2 signaling on podocyte adhesion, we pretreated differentiated ROBO2-overexpressing and vector control mouse podocytes with $10 \mathrm{nM}$ recombinant SLIT2 for 60 minutes. These cells were then seeded onto collagen-coated plates in media containing $10 \mathrm{nM}$ recombinant SLIT2 or buffer as a control for 30 minutes. The nonadherent podocytes were then washed away and the remaining podocytes attached to the collagen were quantified by measuring ATP luminescence signal using the ATPlite assay, a highly sensitive method for the quantification of viable cells (35). In the presence of SLIT2, podocyte attachment to collagen was significantly reduced in both cell lines (Figure 4B), suggesting that exogenous SLIT2 reduces podocyte attachment to collagen. Additionally, TUNEL assays showed that the reduction of viable podocytes attached to collagen upon activation of the SLIT2/ROBO2 pathway was not due to podocyte apoptosis (Supplemental Figure 4). Interestingly, we also observed decreased attachment of ROBO2-overexpressing cells to the matrix without SLIT2 addition. This might be ascribed to paracrine signaling of endogenous SLIT2 activating ROBO2 on the same cells.

To determine if reduced podocyte adhesion is due to an alteration in focal adhesion formation, we performed immunostaining of vinculin and paxillin (Figure 4C), which function as adaptor proteins in focal adhesion formation in podocytes (2). We found that vinculin (Figure 4D) and paxillin (Fig- 
A
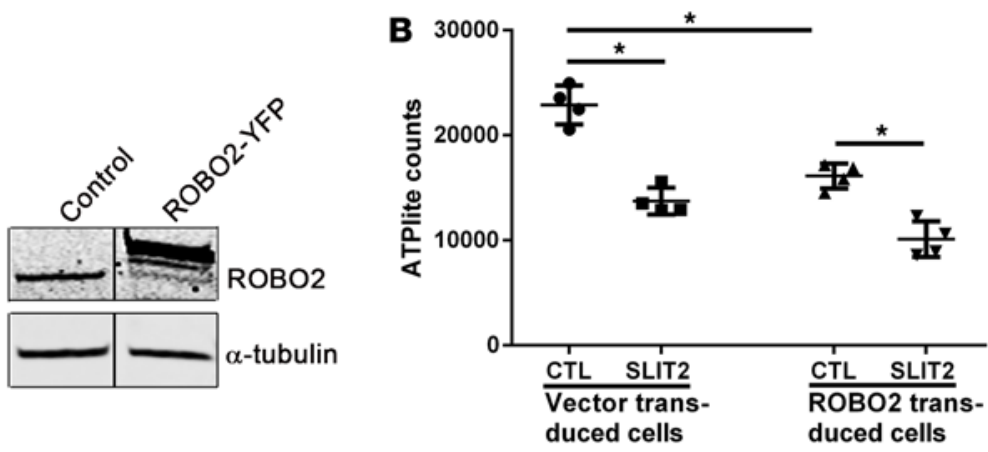

C

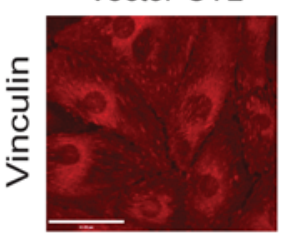

Vector-SLIT2

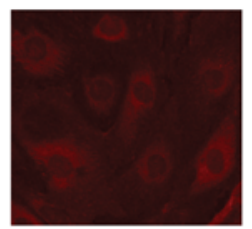

ROBO2-CTL
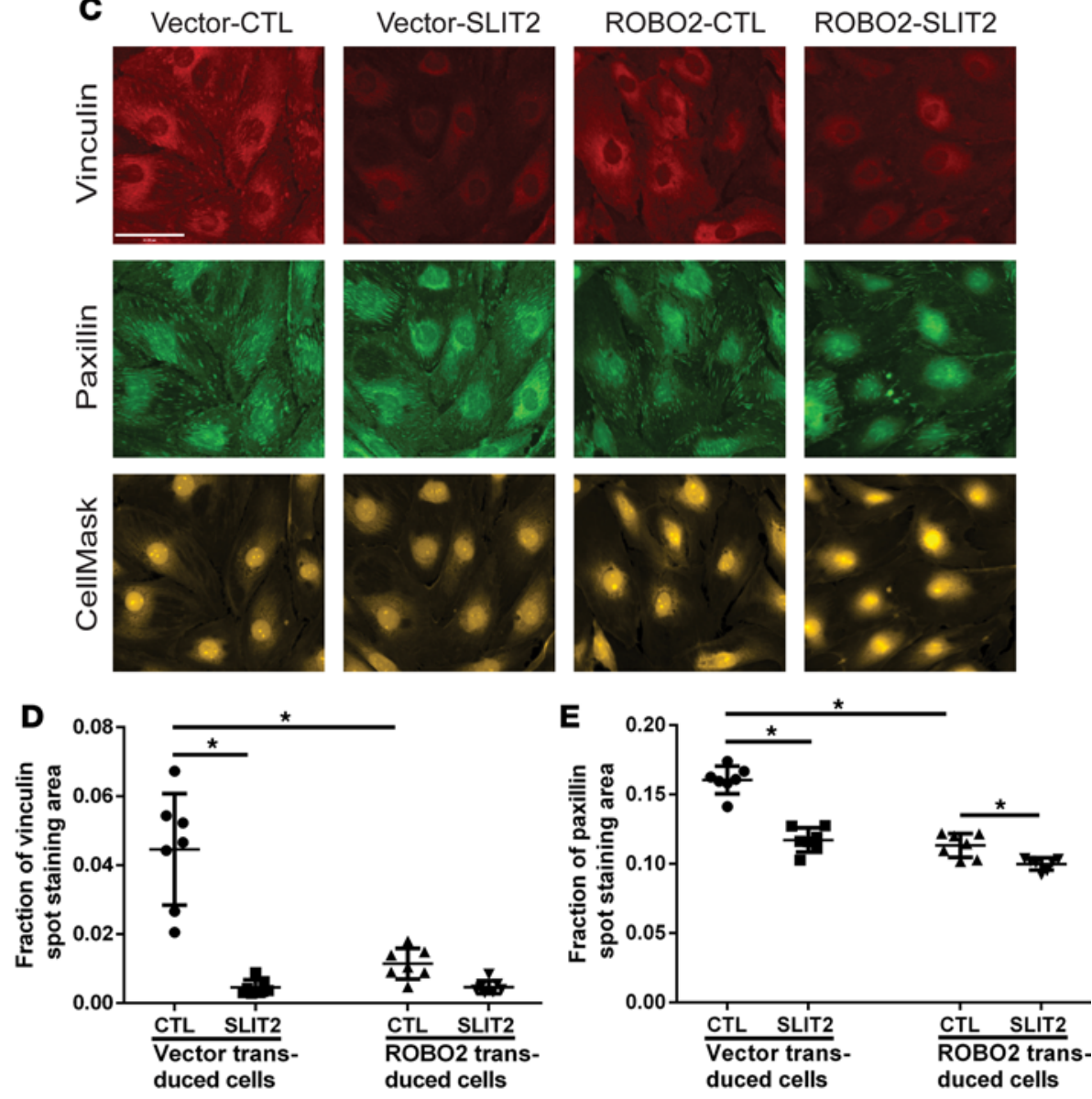

Figure 4. SLIT2-ROBO2 signaling destabilizes podocyte focal adhesions. (A) Expression of ROBO2 in control and ROBO2-YFP overexpressing mouse podocytes detected by Western blot with $\alpha$-tubulin as a loading control. The lanes were run on the same gel but were noncontiguous. (B) Quantification by ATPlite assay of mouse podocytes attached to collagen-coated plates in media containing buffer control (CTL) or $10 \mathrm{nM}$ recombinant SLIT2 N-terminus measured after 30 minutes. Data are presented as the mean $\pm S D, n=4$. ${ }^{*} P<0.05$ compared with the cells as indicated by 1-way ANOVA. Data are representative of at least 3 separate experiments and various time points between 30 and 90 minutes. (C) Representative images of immunostaining of focal adhesion markers vinculin and paxillin in differentiated vector control cells (left 2 columns) or ROBO2-overexpressing mouse podocytes (right 2 columns) treated with buffer (CTL) or SLIT2 N-terminus. The whole cells were labeled by CellMask staining. Scale bar: $61 \mu \mathrm{m}$. (D and $\mathbf{E}$ ) Quantification of vinculin (D) and paxillin (E) spot staining areas divided by the cell spread areas. Data are presented as the mean $\pm \mathrm{SD}, n=7$. ${ }^{*} P<0.05$ compared with the cells as indicated by 1-way ANOVA.

ure $4 \mathrm{E}$ ) spot staining in mouse podocytes was significantly reduced after 1 hour of SLIT2 stimulation. This phenomenon was also observed in podocytes overexpressing ROBO2 (Figure 4, D and E). Taken together, these observations suggest that SLIT2/ROBO2 signaling reduces podocyte focal adhesion formation, which results in decreased cell attachment to collagen-coated surfaces.

Because the cell-cell junction protein $\mathrm{ZO} 1$ is expressed in podocytes and $\mathrm{ZO} 1$ is a known molecular partner of NMIIA (36), we also examined the effect of SLIT2 stimulation on ZO1 distribution in podocytes. Immunostaining of $\mathrm{ZO} 1$ protein showed no changes in $\mathrm{ZO} 1$ dis-

tribution at the cell-cell junctions among vector controls and ROBO2-overexpression podocytes with or without SLIT2 treatment (Supplemental Figure 5), suggesting that SLIT2 stimulation does not impact ZO1-mediated intercellular adhesion. Interestingly, we also observed that SLIT2 can significantly decrease the number of vinculin- and paxillin-positive structures in vector-transfected control podocytes. In addition to the effects from SLIT2/ROBO2 signaling on control podocytes, it is possible that SLIT2 may also have ROBO2-independent effects through other molecules such as plexin A1, a new SLIT2 receptor that also forms a complex with nephrin in podocytes $(37,38)$.

Loss of Robo2 protects mice from hypertension-induced podocyte loss and albuminuria, and also partially rescues podocyte numbers in Myh9 knockout mice. Since SLIT2/ROBO2 inhibits NMII activity and reduces adhesion of cultured podocytes, we asked if ROBO2 has the same biological effect on NMII in podocytes in vivo. To test this hypothesis, we generated podocyte-specific Robo2 and Myh9 (encodes NMHC IIA) single conditional knockout (cKO) mice and Robo2-Myh9 double conditional knockout mice using Nphs2-Cre. As previously reported $(7,30)$, neither Robo2 single cKO $\left(\right.$ Robo $\left.2^{\mathrm{f} / \mathrm{fl}} ; N p h s 2-C r e^{+}\right)$, nor Myh9 single cKO $\left(M y h 9^{\mathrm{f} / \mathrm{fl}} ; N p h s 2-C r e^{+}\right)$, 
A

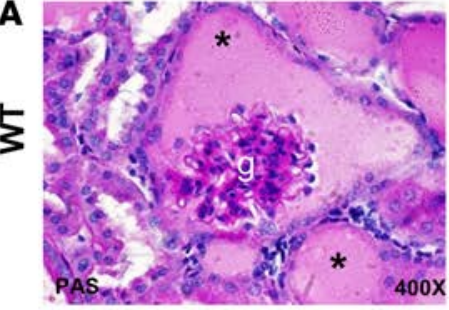

C
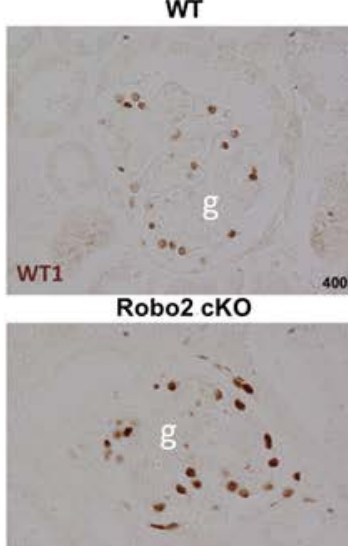

WT1

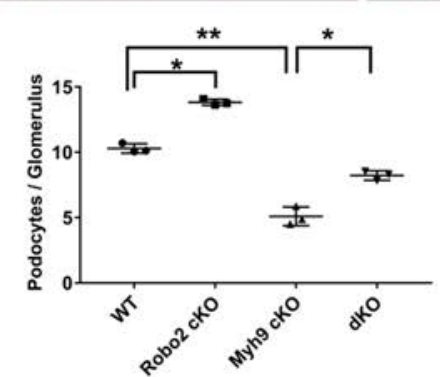

\section{D}
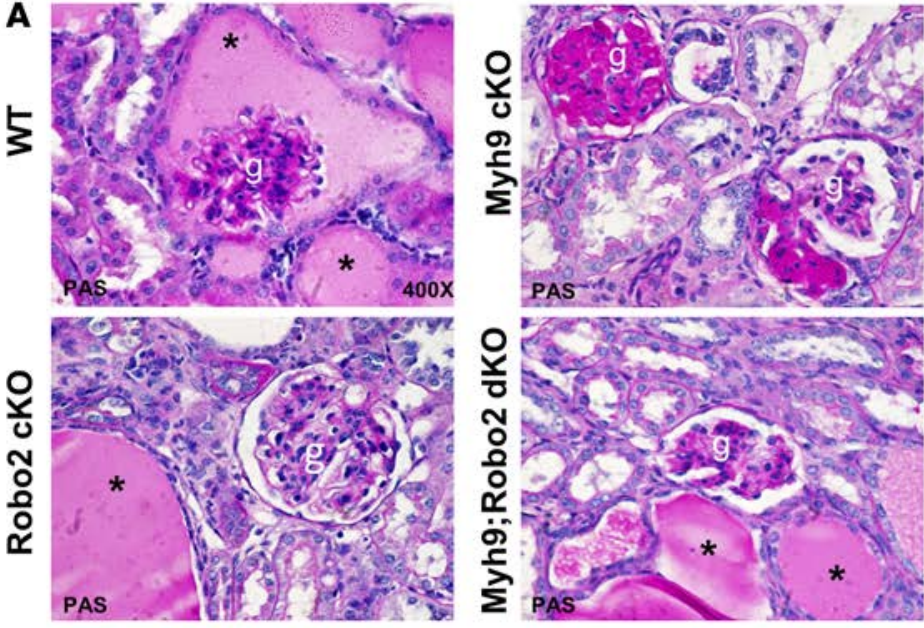

Myh9 cKO

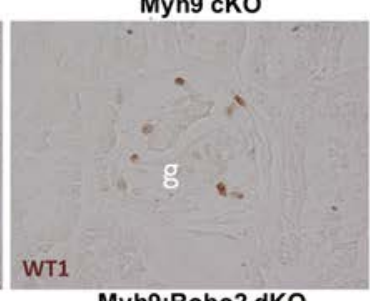

Myh9;Robo2 dKO

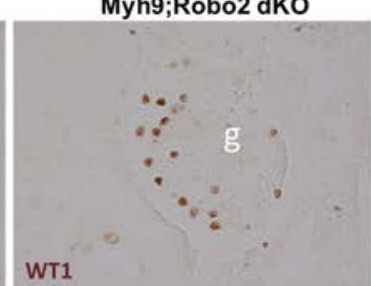

Figure 5. Robo2 deficiency protects mice from DOCA-salt-uninephrectomy-induced podocyte loss. (A) Representative images of periodic acid-

Schiff (PAS) staining of mouse kidney tissues show proteinaceous casts (asterisks), glomerulosclerosis, and increased matrix expression after 5-week deoxycorticosterone acetate (DOCA)-salt-uninephrectomy hypertension injury in wild-type, Myh9 conditional knockout (cKO), Robo2 cKO, and Myh-Robo2 double KO mice. Magnification: $\times 400$. g, glomerulus. (B) Quantification of percentage of sclerotic glomeruli in kidney samples from A. Data are presented as the mean \pm SEM, $n=3$ animals per group, minimum 100 glomeruli counted in each animal. $P=0.028$ compared with the Myh9 cKO group by 1 -way ANOVA. (C) Representative images of WT1-positive podocytes in the glomeruli isolated from 4 groups of mice with Myh9/Robo2 single cKO, Myh9-Robo2 double cKO (dKO), and wild-type (WT) genotype after 5-week DOCA-salt-uninephrectomy injury. Magnification: $\times 400$. g, glomerulus. (D) Quantification of podocyte number per glomerulus. Data are presented as the mean $\pm \mathrm{SEM}, n=3$ animals per group, minimum 50 glomeruli counted in each animal. ${ }^{*} P<0.05,{ }^{*} P<0.01$ by 1-way ANOVA. (E) Albuminuria from the mice 5 weeks after DOCA-salt-uninephrectomy injury detected by acrylamide gel and

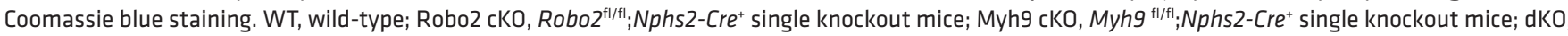

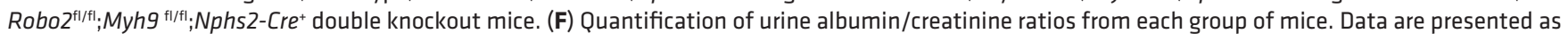
the mean $\pm \mathrm{SEM}, n=4$ animals. ${ }^{*} P<0.01,{ }^{* *} P<0.05$ by 1 -way ANOVA.

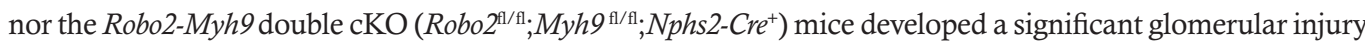
phenotype, as all single or double homozygous $\mathrm{cKO}$ mice have a normal renal histology without proteinuria (Supplemental Figure 6). To determine if deletion of $M y h 9$ and Robo2 affects podocyte number, we stained glomeruli for WT1 and counted the number of WT1-positive podocytes in each group. We observed that 

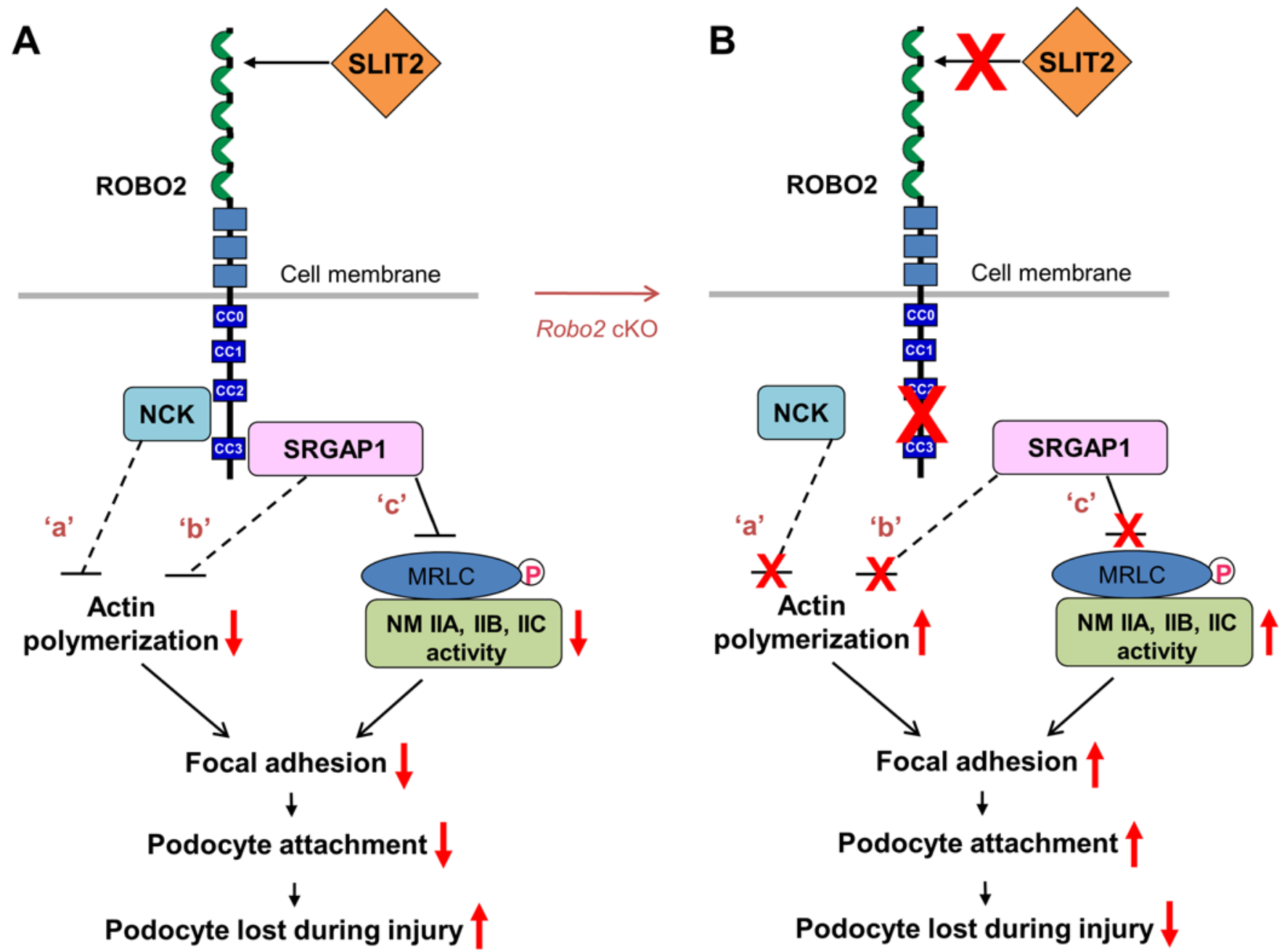

Figure 6. The proposed model: SLIT2/ROBO2 signaling destabilizes podocyte adhesion and attachment. (A) ROBO2 is predominantly localized on the basal membrane of podocytes, and SLIT2 is secreted by podocytes (7) (also see Supplemental Figure 3). Upon SLIT2 binding, ROBO2 increases its binding to adaptor protein NCK (7) which, in turn, interacts with N-WASP and Arp2/3 to inhibit actin polymerization (62) (signaling 'a'). ROBO2 also recruits SRGAP1, which inactivates the small GTPase Cdc42 to block actin polymerization (14) (signaling ' $b$ '). The SH3 domain of SRGAP1 binds to ROBO2, and its F-BAR domain is associated with nonmuscle myosin IIA (NMIIA)/IIB/IIC through myosin regulatory light chain (MRLC). The complex of ROBO2/SRGAPs/ MRLC/NMII results in inactivation of NMII, possibly due to structurally blocking MRLC phosphorylation (signaling ' $c$ '). F-actin polymerization and NMIIA activity are required for normal focal adhesion formation and maturation $(2,26)$. Inactivation of actin polymerization and inhibition of NMII might interfere with focal adhesion assembly and result in a decrease in podocyte attachment. Signaling 'a': published data from Fan et al. (7); signaling 'b': published data from Wong et al. (14); signaling 'c': data from this study. Dashed lines indicate indirect inhibition while the solid line shows the direct inhibition. Red arrows indicate enhanced or inhibited activities. CCO-3, cytoplasmic conserved regions 0-3. (B) Removal of the SLIT2/ROBO2 inhibitory signaling in podocytes (e.g., in Robo2 cKO mice) promotes focal adhesion formation and podocyte attachment, which protects against podocyte loss during injury.

Myh9 single cKO mice have fewer podocytes compared with their wild-type littermates (Supplemental Figure 7). Interestingly, the Robo2-Myh9 double cKO mice have a comparable number of podocytes relative to their wild-type littermates, suggesting that loss of Robo2 in podocytes might rescue the podocyte loss induced by Myh9 deletion.

Because deficiency of Myh9 and/or Robo2 in podocytes did not cause striking glomerular phenotypes, we chose to challenge these mice by inducing glomerular capillary hypertension in uninephrectomized mice with deoxycorticosterone acetate (DOCA) combined with high-salt drinking water to enhance podocyte injury $(39,40)$. DOCA is a precursor for aldosterone that plays a critical role in regulating sodium and water retention in the renal distal tubules and collecting ducts to control blood pressure. With increased salt in the drinking water, combined with a reduction in nephron number caused by uninephrectomy, DOCA-salt-uninephrectomy causes hyperaldosteronism, hypervolemia, and salt-sensitive hypertension in mice $(39,40)$. In this model, high glomerular capillary pressure increases the tension of the glomerular 
capillary wall, leading to podocyte injury, detachment, albuminuria, and subsequent glomerulosclerosis (41). Although all groups of mice developed hypertension after DOCA-salt-uninephrectomy injury (Supplemental Figure 8), the glomerular phenotype appeared to be less severe in those mice that were deficient in podocyte Robo2 by either periodic acid-Schiff (PAS) staining or electron microscopy (Figure 5, A and B, and Supplemental Figure 9). To evaluate podocyte loss, we counted the number of WT1-positive podocytes in injured mice after 5 weeks. Notably, Robo2 single cKO mice had the highest number of podocytes compared with the $M y h 9$ single cKO mice and wild-type littermate controls (Figure 5, C and D). Interestingly, Robo2-Myh9 double cKO mice had significantly more podocytes as compared with the Myh9 single cKO mice, suggesting that loss of Robo2 partially rescued podocyte loss in the Myh 9 CKO (Figure 5 , $\mathrm{C}$ and D). This result is also confirmed by P57 staining, a podocyte-specific marker without labeling the glomerular parietal epithelial cell (Supplemental Figure 10) $(42,43)$. Consistent with the podocyte-loss phenotype, DOCA-salt-uninephrectomy injury also caused severe albuminuria in the wild-type and Myh 9 single cKO mice. By contrast, both Robo2 single cKO mice and Robo2-Myh9 double cKO mice developed significantly less proteinuria (Figure 5, E and F, and Supplemental Figure 11). Taken together, these results suggest that loss of Robo2 protects mice from hypertension-induced podocyte loss and also partially rescues podocyte numbers in the Myh9 single knockout mice.

\section{Discussion}

SLIT is a repulsive guidance cue that negatively regulates the cytoskeleton of the growth cone to repel navigating axons and migrating neurons (34). In migrating neurons, upon SLIT binding, ROBO receptors recruit SRGAPs to inactivate Cdc42, which in turn decreases actin polymerization (14). SLIT2/ROBO2 signaling has recently also been shown to inhibit myosin II activity in Schwann cells, but the signaling pathway has not been studied (44). We previously reported that the SLIT2/ROBO2 signaling pathway regulates podocyte structure and function by inhibiting nephrin-associated actin polymerization through the adaptor protein NCK (7). In this study, we found that SRGAP1 interacts with MRLC, a subunit of NMII, and forms a complex with ROBO2 and NMHC IIA. Since ROBO2 and SRGAP1 are uniquely expressed in the podocytes in the kidney, and MRLC and NMHC IIA are also expressed in these cells in the kidney, our results indicate that ROBO2, SRGAP1, MRLC, and NMHC IIA are able to form a complex in podocytes. This complex formation is also supported by the subcellular colocalization of ROBO2 and SRGAP1 at the basal membrane of the podocyte, while NMHC IIA is also predominantly localized to the leading area of the cell membrane (26). Our study thus identified SRGAP1 as a potentially novel link between SLIT2/ROBO2 signaling and NMII activity in the podocyte.

The activity of NMII, including NMIIA, NMIIB, and NMIIC, plays a central role in the control of cellular shape, cell adhesion, and cell migration through its actin cross-linking and contractile functions (26). This NMII activity is regulated by the phosphorylation of its regulatory light chain, MRLC (26). Our study demonstrates that the 172-amino acid MRLC subunit interacts directly with SRGAP1 and this interaction is independent of its phosphorylation. Podocytes treated with SLIT2 show decreased phosphorylation of MRLC, suggesting that SLIT2/ROBO2 signaling inhibits NMII activation. Although the exact mechanism of MRLC dephosphorylation by SLIT2/ROBO2 signaling remains to be determined, one possibility is that the association of SRGAP1 and nonphosphorylated MRLC might block the kinases that bind and phosphorylate MRLC, resulting in NMII inactivation. In addition, SLIT2/ROBO2 signaling might inhibit NMII activity by recruiting MRLC to the membrane area through SRGAP1 and reducing MRLC phosphorylation via myosin phosphatase (e.g., PPP1R12A/ MYPT1), which is abundant in the membrane area.

The function of the blood-urine barrier relies on podocyte adhesion both at cell junctions (i.e., the slitdiaphragm) and at the interface between the basal surface of the podocyte and the extracellular matrix of the GBM. Podocytes attach to the basement membrane through focal adhesion formation which requires NMII activity for assembly of actin bundles and F-actin cytoskeleton connection (2, 26). NMII activation promotes adhesion maturation, which results in the formation of large actin bundles and stable adhesions between podocytes and the GBM (26). Impairing or decreasing NMII activity will reduce actin bundling and podocyte adhesion, which leads to podocyte detachment and loss during injury. Because NMII influences cell adhesion from a distance by attaching to the actin bundles through which cell adhesions are associated, SLIT2/ROBO2 signaling reduces podocyte adhesion and promotes podocyte loss during injury, which may be ascribed to combined effects of decreasing actin polymerization (signaling ' $a$ ' and 'b' 
in Figure 6A) and inhibiting NMII activity (signaling 'c' in Figure 6A) (7, 14, 26).

Our in vivo animal model study showed that ablation of NMIIA in podocytes (i.e., Myh9 single cKO) decreases total glomerular podocyte number, but loss of both NMIIA and ROBO2 (i.e., Robo2-Myh9 double cKO) partially rescues the podocyte-loss phenotype. Since SLIT2/ROBO2 signaling inhibits NMII activity, which regulates cellular focal adhesion formation and F-actin organization (26), abolition of ROBO2 likely reduces the inhibitory effect on NMII and increases overall NMII activity generated by NMIIA, NMIIB, and NMIIC. In the absence of both NMIIA and ROBO2 (i.e., Robo2-Myh9 double cKO), NMIIB (encoded by the Myh10 gene) and NMIIC (encoded by the Myh14 gene) could have increased activity to compensate for the loss of NMIIA and increase overall NMII activity and promote podocyte adhesion. This might explain the alleviated podocyte-loss phenotype in the Robo2-Myh9 double cKO mice. In addition, SLIT2/ ROBO2 signaling also inhibits actin polymerization, and deletion of ROBO2 will likely increase actin polymerization and focal adhesion formation. Taken together, our results show that removal of SLIT2/ ROBO2 inhibitory signaling in podocytes (e.g., in Robo2 cKO) promotes focal adhesion formation and podocyte attachment, which limits podocyte loss during injury (Figure 6B).

Podocyte detachment and loss lead to proteinuria and glomerulosclerosis, the morphological hallmark of many chronic kidney diseases (45-51). Podocyte injury usually begins with foot process effacement and is followed by detachment from the GBM (52). Early protection from podocyte detachment and loss is important and renoprotective because local podocyte injury and loss can trigger secondary damage of neighboring intact podocytes, leading to a vicious cycle of accelerated podocyte loss, proteinuria, and glomerulosclerosis $(53,54)$. Our studies suggest that the SLIT2/ROBO2 signaling pathway might be a therapeutic target for renoprotection. Blocking this pathway may prevent and reduce podocyte detachment and loss, which in turn will enhance podocyte survival and restore the glomerular filtration barrier in patients with chronic kidney disease.

\section{Methods}

Yeast 2-hybrid assay. The DupLEX-A yeast 2-hybrid system (OriGene Tech) was used to characterize ROBO2, SRGAP1, and MRLC interactions as previously reported (7). The cDNAs encoding the intracellular domain of human ROBO2 and its truncated forms were subcloned into the pJG4-5 vector at the EcoRI/XhoI sites, fusing them to the transcription activation domain of B42. The cDNAs of the SH3 domain of human SRGAP1 was subcloned into the pEG202 vector at EcoRI/XhoI to fuse them to the DNA-binding domain of LexA. To test the interaction of SRGAP1 and MRLC, the F-BAR domain of SRGAP1 was fused to LexA while MRLC and its mutants were fused to the B42 domain. The lacZ gene in the construct pSH18-34 and the LEU2 gene in the EGY48 strain yeast genome were used as reporter genes. The pEG202, pSH18-34, and pJG4-5 constructs were cotransformed into yeast EGY48 cells. The interaction was considered positive if the yeast cells turned blue in the presence of X-gal and grew in the absence of leucine.

Constructs with mutated MRLC phosphorylation sites were made by replacing the threonine and serine at positions 18 and 19 with alanine or aspartic acids, respectively. MRLC cDNA was subcloned into the pJG4-5 vector at the EcoR1/Xho1 sites. Single amino acid substitution was performed using the QuickChange Site-Directed Mutagenesis Kit (Stratagene) according to the manufacturer's instructions.

Coprecipitation and Western blot analyses. HEK cells were transfected at $60 \%$ confluence using calcium phosphate transfection. To make C-terminal His- and myc-tagged fusion proteins, full-length human ROBO2 and SRGAP1 were subcloned into the pSecTag B vector (Invitrogen) at the EcoR1/Xho1 restriction sites. SRGAP1- $\triangle \mathrm{F}-\mathrm{BAR}$ was made by deleting the $\mathrm{N}$-terminal F-BAR binding domain using the QuikChange Site-Directed Mutagenesis Kit according to the manufacturer's protocol. To test the SRGAP1 and NMIIA interaction, C-terminal His- and myc-tagged human SRGAP1 or SRGAP1$\triangle$ F-BAR was expressed in HEK cells. Forty-eight hours after transfection, cells were lysed in $50 \mathrm{mM}$ $\mathrm{NaH}_{2} \mathrm{PO}_{4}, 300 \mathrm{mM} \mathrm{NaCl}, 10 \mathrm{mM}$ imidazole, $0.5 \%$ Triton $\mathrm{X}-100$, and $1 \times$ protease inhibitor cocktail $(\mathrm{pH}$ 8.0). Cell lysates were centrifuged for 10 minutes at $4^{\circ} \mathrm{C}$, and supernatants were incubated with Ni-NTA resin (Qiagen) at $4^{\circ} \mathrm{C}$ for 2 hours to precipitate His-ROBO2, and NTA resin without Ni was used as a control. The resin was washed 3 times with washing buffer $\left(50 \mathrm{mM} \mathrm{NaH} \mathrm{PO}_{4}, 300 \mathrm{mM} \mathrm{NaCl}, 20\right.$ $\mathrm{mM}$ imidazole, $0.5 \%$ Triton $\mathrm{X}-100[\mathrm{pH} 8.0])$ and heated at $95^{\circ} \mathrm{C}$ for 10 minutes. The precipitates were resolved in SDS-PAGE gels and blotted with rabbit anti-myc (Sigma-Aldrich, C3956), rabbit monoclonal anti-NMHC IIA (Cell Signaling Technology, 3403), and anti-MRLC (Sigma-Aldrich, MY-21) antibodies 
at 1:1,000 dilution. To examine the triple interaction among ROBO2, SRGAP1, and NMIIA, His-mycROBO2 or His-myc-ROBO2- $\triangle \mathrm{CC} 3$ was expressed in HEK cells. His-myc-ROBO2 was precipitated with Ni-NTA beads as described above. Precipitates were blotted with rabbit polyclonal anti-myc, rabbit anti-SRGAP1 (Novus Biologicals, NB100-60650), rabbit anti-NMHC IIA, and mouse monoclonal antiMRLC antibodies at 1:1,000 dilution. For coimmunoprecipitation of endogenous proteins, newborn mouse kidneys were homogenized in RIPA buffer (50 mM Tris [pH 7.4], $150 \mathrm{mM} \mathrm{NaCl}, 0.1 \%$ SDS, $1 \%$ NP-40, $0.5 \%$ sodium deoxycholate, $1 \mathrm{mM} \mathrm{Na}_{3} \mathrm{VO}_{4}, 1 \mathrm{mM} \mathrm{NaF}$, and $1 \times$ protease inhibitor cocktail) on ice. Samples were centrifuged for 10 minutes at $4^{\circ} \mathrm{C}$, and the supernatants were incubated with $1 \mu \mathrm{g}$ mouse monoclonal anti-ROBO2 antibody (R\&D Systems, MAB3147) for 1 hour at $4^{\circ} \mathrm{C}$. Goat IgG (Santa Cruz Biotechnology) was used as a control. Samples were then mixed with $30 \mu 1$ of protein A/G Plus agarose bead slurry (Santa Cruz Biotechnology) and further incubated for 12 hours at $4^{\circ} \mathrm{C}$. Beads were then washed 3 times in RIPA buffer, and proteins were eluted in $1 \times$ protein loading buffer by heating them at $95^{\circ} \mathrm{C}$ for 10 minutes. Precipitates were resolved in SDS-PAGE gels and blotted with mouse anti-ROBO2, rabbit anti-SRGAP1, rabbit anti-MRLC, anti-p-MRLC (Thr18/Ser19) (Cell Signaling Technology, 3674), and anti-NMHC IIA antibodies as described above.

Immunofluorescence staining. Immunofluorescence staining was performed on mouse kidney tissues fixed in $4 \%$ paraformaldehyde as previously described (7). Briefly, fixed mouse kidneys were embedded in OCT compound and cut into 8- to $10-\mu \mathrm{m}$ sections on a cryostat. Sections were stained with primary antibodies followed by appropriate Alexa Fluor 488- or Cy3-conjugated secondary antibodies. The primary antibodies used in this study include anti-ROBO2 (Santa Cruz Biotechnology, sc-31607), anti-SRGAP1 (Santa Cruz Biotechnology, sc-81939), anti-nephrin (custom synthesized) (55), anti-podocin (Sigma-Aldrich, P0372), antiSLIT2 (Santa Cruz Biotechnology, sc-26601), anti-ZO1 (Proteintech, 217731AP), anti-MRLC (Sigma-Aldrich, MY-21), and anti-NMHC IIA (Cell Signaling Technology, 3403). Images were obtained using a Nikon deconvolution wide-field epifluorescence system and a Zeiss LSM 710 confocal laser scanning microscope.

Generation of stable human ROBO2-YFP-overexpressing mouse podocytes. The lentiviral expression vector from Biosettia, pLV-EF1 $\alpha$-MCS-IRES-Puro (catalog cDNA-pLV01), was used to subclone human ROBO2 cDNA fused with a YFP tag at the $3^{\prime}$ end. Plasmid DNA and ViraPower Lentiviral Packaging Mix (catalog K4975-00) were transfected into 293T cells to package lentiviral particles following the manufacturer's protocol. Supernatants containing lentiviral particles were collected 48 hours after plasmid transfection. Lentivirus-containing supernatants $(200 \mu \mathrm{l})$ were utilized to infect $4 \times 10^{4}$ podocytes. Human ROBO2overexpressing stable cells were selected by $1 \mu \mathrm{g} / \mathrm{ml}$ puromycin in mouse podocyte growth media (RPMI containing $10 \% \mathrm{FBS}$ and $10 \mathrm{U} / \mathrm{ml} \mathrm{IFN}-\gamma$ (Invivogen, catalog CR2041) and cultured at $33^{\circ} \mathrm{C}$ until mock-infected cells died. Expression of human ROBO2 was confirmed by Western blotting and immunofluorescence staining (data not shown). Vector control podocytes were similarly generated by infecting mouse podocytes with lentiviral particles containing an empty vector.

Podocyte and SVZa coculture neuronal migration assay. The in vitro neuronal migration assay using postnatal SVZa cells was described previously (56). Briefly, P1 Sprague-Dawley rat brains devoid of meninges were placed in DMEM with $10 \%$ fetal calf serum. Coronal sections of the SVZa brain tissues were dissected by vibratome to make SVZa explants of 200 - to $300-\mu \mathrm{m}$ diameter. To make coculture cell aggregates, HEK cells were transiently transfected to express human SLIT2 or control vectors as negative controls using the Effectene Transfection Kit (Qiagen). After 24 hours, transfected HEK cells, as well as cultured podocytes (nondifferentiated and differentiated), were collected by brief centrifugation, and cell pellets were resuspended in an equal volume of DMEM. Suspended cells $(10 \mu \mathrm{l})$ were hung from the dish cover at $37^{\circ} \mathrm{C}$, in $5 \% \mathrm{CO}_{2}$ for 1 to 2 hours to form cell aggregates. Aggregated cells were washed in DMEM and squared with a needle. SVZa explants were then embedded together with the cell aggregates in collagen and matrigel (3:2:1 collagen/matrigel/medium) and cocultured in DMEM at $37^{\circ} \mathrm{C}, 5 \% \mathrm{CO}_{2}$ for 24 hours . Cocultured cells were washed in PBS for 10 minutes and fixed in $4 \%$ paraformaldehyde at $4^{\circ} \mathrm{C}$ overnight. The fixed SVZa neuronal cells were then imaged and cell migration was analyzed.

Podocyte culture and MRLC phosphorylation assays. Immortalized mouse podocytes (33) (provided by Jochen Reiser, Rush University Medical Center, Chicago, Illinois, USA) suspended in RPMI 1640 media containing $10 \%$ FBS were plated onto collagen I-coated 6 -well plates and differentiated at $37^{\circ} \mathrm{C}$ in a cell culture incubator for 7 days. Cells were then treated with $50 \mathrm{nM}$ recombinant human SLIT2 N-terminus (SLIT2-N) (Sigma-Aldrich, SRP3155) or buffer for 10, 60, and 120 minutes. After removing the media, cells were lysed in $25 \mathrm{mM}$ Tris- $\mathrm{HCl} \mathrm{pH}$ 7.4, $150 \mathrm{mM} \mathrm{NaCl}, 1 \%$ NP-40, $1 \mathrm{mM}$ EDTA, 5\% glycerol. Cell 
lysates were then loaded onto NuPAGE Bis-Tris precast gels (Life Technologies) followed by protein transfer onto nitrocellulose membranes by using the iBlot2 system (Life Technologies). Total MRLC and p-MRLC were measured by blotting with antibodies against MRLC (Cell Signaling Technology, 8505) and p-MRLC (Thr18/Ser19) (Cell Signaling Technology, 3674), respectively, followed by detection with a IRDye 800CW-conjugated secondary antibody (LI-COR, 926-32213). Blots were scanned by the Odyssey Infrared Imaging System (LI-COR Biosciences). Ratios of the fluorescence intensity of p-MRLC to that of total MRLC were quantified by ImageJ software (NIH).

Podocyte adhesion assay. Mouse podocytes in RPMI 1640 media containing 10\% FBS were plated onto collagen-coated $10-\mathrm{cm}$ dishes and differentiated at $37^{\circ} \mathrm{C}$ in a cell culture incubator for 7 days. Cells were then pretreated with $10 \mathrm{nM}$ SLIT2-N or buffer in RPMI 1640 media containing 1\% FBS for 1 hour followed by dissociation from plates with Accutase solution (Sigma-Aldrich, A6964). Dissociated mouse podocytes were then plated into 2 replicated collagen I-coated 96-well plates in media containing 1\% FBS and $10 \mathrm{nM}$ SLIT2-N or buffer. To quantify and normalize the number of cells seeded in each well, 1 plate was analyzed using the ATPlite 1Step kit (PerkinElmer, catalog 6016731) immediately after the cells were plated. This assay generates luciferase activity as a function of cell number (35). The other plate was incubated at $37^{\circ} \mathrm{C}$ for 30 minutes to allow the cells to adhere. After incubation, the podocytes were gently washed twice with PBS to remove the nonadherent cells. The adherent cells were subjected to the ATPlite assay and luminescence was measured. The ATPlite counts generated by the adherent cells were normalized to the initial ATPlite counts to control for potential unequal initial cell seeding. The adhesion assays were repeated several times at initial cell seedings of 2,000 to 6,000 podocytes/well.

Immunocytochemistry of vinculin and paxillin in mouse podocytes. Mouse podocytes suspended in RPMI 1640 media containing 10\% FBS were plated onto collagen I-coated 96-well plates and differentiated at $37^{\circ} \mathrm{C}$ in a cell culture incubator for 7 days. Cells were then treated with $10 \mathrm{nM}$ recombinant SLIT2-N or buffer in 1\% FBS RPMI 1640 media for 1 hour and then fixed with 4\% paraformaldehyde at room temperature for 30 minutes. Cells were washed twice with PBS and then blocked with 5\% goat and 5\% donkey serum for 1 hour at room temperature. Mouse podocytes were stained with anti-vinculin (SigmaAldrich, V-9131) and anti-paxillin (Millipore, 04-581) antibodies at room temperature for 1 hour. After washing, cells were incubated with secondary antibodies conjugated with fluorophores followed by nuclear staining with Hoechst 33342 (Life Technologies, H3570) and whole-cell staining with HCS CellMask Orange Stain (Life Technologies, H32713). Immunostained cells were scanned using the Operetta Imaging System (PerkinElmer). Vinculin and paxillin staining was quantified by measuring the areas of vinculin or paxillin staining spots. The fraction of vinculin or paxillin spot staining was calculated by dividing the sum of vinculin or paxillin spot staining areas within a cell by the area of the whole cell. The mean of more than 300 cells was analyzed and compared.

Podocyte apoptosis assay. Podocyte apoptosis was measured using a Click-iT TUNEL Alexa Fluor Imaging Assay (Thermo Fisher, C10245) according to the manufacturer's directions. Briefly, differentiated mouse podocytes were fixed using 4\% paraformaldehyde in PBS for 15 minutes at room temperature followed by permeabilization in $0.25 \%$ Triton X-100 at room temperature for 20 minutes. Podocytes were then washed twice with deionized water. DNase treatment of a few wells was used as a control to create strand breaks in the DNA to provide a positive TUNEL reaction. Podocytes were incubated with DNase I for 30 minutes at room temperature and then washed with deionized water. The cells were equilibrated with terminal deoxynucleotidyl transferase (TdT) reaction buffer for 10 minutes and then incubated with the TdT reaction cocktail for 1 hour at $37^{\circ} \mathrm{C}$. Cells were then washed twice with $3 \%$ BSA in PBS, incubated with the Click-iT reaction cocktail for 30 minutes at room temperature in the dark, and washed in 3\% BSA in PBS. Finally, nuclei were stained with Hoechst 33342. The cells were imaged on the Operetta Imaging System using identical exposure settings across all conditions.

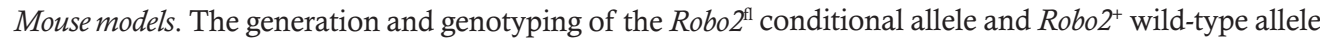
were described previously (20). The $M y h^{\natural}$ conditional allele was published previously (57) and was obtained from the EMMA mouse repository (EMMA ID: EM02572). Standard breeding schemes were followed to

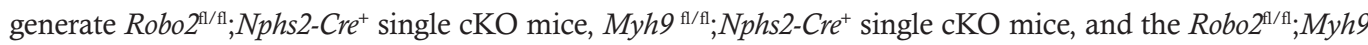
${ }_{\mathrm{fl} / \mathrm{fl}} ; \mathrm{Nphs} 2-\mathrm{Cre}^{+}$double $\mathrm{cKO}$ mice. A Srgap1-lacZ mouse reporter line was generated by in vitro fertilization using cryopreserved sperm obtained from the KOMP repository (ID: CSD36665) in the transgenic core at Boston University Medical Center. $\beta$-Galactosidase staining of Srgap1-lacZ reporter mouse embryonic kidneys was performed according to established methods (58). Briefly, Srgap1-lacZ kidneys were harvested 
and placed in ice-cold PBS, followed by fixation in $0.2 \%$ glutaraldehyde for 30 minutes. The kidneys were then incubated overnight at $4^{\circ} \mathrm{C}$ in $\beta$-galactosidase staining solution ( $\beta$-galactosidase substrate $0.25 \mathrm{mg} / \mathrm{ml}$ ), $2 \mathrm{mM} \mathrm{MgCl}_{2}, 0.01 \%$ Na-deoxycholate, $0.02 \% \mathrm{NP}-40,5 \mathrm{mM} \mathrm{K}_{4} \mathrm{Fe}(\mathrm{CN})_{6}$, and $5 \mathrm{mM} \mathrm{K}_{3} \mathrm{Fe}(\mathrm{CN})_{6}$.

DOCA-salt-uninephrectomy injury model. The DOCA-salt-uninephrectomy injury model was performed according to standard published methods $(40,59)$. Briefly, 10 -week-old Robo2 $2^{\mathrm{f} / \mathrm{fl}} ; \mathrm{Nphs} 2-\mathrm{Cr} \mathrm{e}^{+}$single cKO

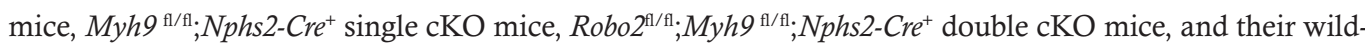
type littermate controls ( $n=3$ in each group) underwent left unilateral nephrectomy after anesthesia. Mice were then implanted with a 60-day, slow-release pellet containing $150 \mathrm{mg}$ of DOCA (Innovative Research of America) under the back skin of the neck. All animals were fed a standard rodent diet and were provided with isotonic saline as drinking water $(0.9 \% \mathrm{NaCl})$. Blood pressure was measured 3 times per week by the noninvasive tail-cuff method (BP-2000 Blood Pressure Analysis System, Visitech Systems, Inc.). Urine samples were collected every week for 5 weeks. After 5 weeks, animals were euthanized, and the kidneys were collected and fixed in formalin overnight. Tissues were dehydrated and paraffin embedded and cut into 5 - $\mu$ m sections.

Mouse kidney pathology studies, quantification of podocytes, and proteinuria analysis. For kidney pathological studies, mouse kidneys were dissected and fixed in formalin or 4\% paraformaldehyde overnight and then treated with a graded ethanol series for paraffin embedding. The kidney paraffin blocks were cut into 5 - $\mu \mathrm{m}$ sections using an MT-920 microtome (MICROM) and stained using standard PAS or H\&E methods. The kidneys were examined and assessed for glomerular matrix deposition, segmental glomerulosclerosis, dilatations of the renal tubules, and proteinaceous casts using an Olympus BHT light microscope equipped with a SPOT digital camera system. For quantification of podocytes, WT1 or P57 was used as a podocyte nuclear marker and immunoperoxidase staining was performed on kidney sections following a protocol described previously (60). Briefly, kidney tissue sections were immunostained according to the Vectastain Elite ABC Kit (Vector Laboratories) protocol with minor modifications. Paraffin-embedded tissue sections were deparaffinized and hydrated through xylene and with graded alcohol. Antigen retrieval was performed in $0.1 \mathrm{M}$ EDTA/0.05\% Tween 20 buffer in a steamer for 45 minutes. After 3 washes with PBS/Tween 20, sections were blocked for 1 hour at room temperature with goat serum. After a brief rinse, sections were incubated with either rabbit anti-WT1 antibody (Abcam, ab89901) at a 1:300 dilution or rabbit anti-P57 antibody (Santa Cruz Biotechnology, sc-8298) at a 1:700 dilution overnight at $4^{\circ} \mathrm{C}$ and then with goat anti-rabbit biotinylated secondary antibody and $\mathrm{ABC}$ solution (Vector Laboratories) according to the manufacturer's instructions. ImmPACT DAB (Vector Laboratories) was used as a chromogenic substrate. Tissue sections were dehydrated by an alcohol gradient and xylene and mounted with Permount (Electron Microscopy Sciences, 17986-01). Podocytes in each glomerulus were counted under the $40 \times$ objective and 50 glomeruli were counted in each animal. Kidney images were obtained using a light microscope with a $40 \times$ oil immersion objective. For proteinuria analysis, spot urine specimens were collected and analyzed by Coomassie blue staining assay according to a published method (61). Briefly, equal volumes of diluted spot urine samples were run in SDS-PAGE gels along with $2 \mathrm{mg}$ albumin standard followed by Coomassie Brilliant Blue staining. The Coomassie-stained gels were quantified by densitometric measurements using NIH ImageJ software. The densitometric units of the urine samples were converted into urinary albumin/ creatinine ratios after their normalization to urinary creatinine values and the dilution factor using a calibration curve.

Transmission electron microscopy. Small kidney pieces from the midsection of the mouse kidney were fixed in $3 \%$ glutaraldehyde-based fixative overnight at $4^{\circ} \mathrm{C}$. The tissue was postfixed in $1 \%$ osmium tetroxide, dehydrated in graded alcohols, and embedded in epoxy resin. Semithin sections ( $2 \mu \mathrm{m}$ thick) were cut using a glass knife on a Leica UC7 microtome and stained with toluidine blue dye. Ultrathin sections $(0.1 \mu \mathrm{m}$ thick) were prepared and stained with $4 \%$ aqueous uranyl acetate and $0.4 \%$ lead citrate. Tissue grids were then examined with a JEOL JEM-1011 transmission electron microscope, and detailed electron microscopic images of the glomeruli were taken with a digital camera system (Gatan Erlangshen ES1000W).

Statistics. A minimum of 3 mice were used for each analysis unless stated otherwise. Data are presented as the mean \pm SEM or SD. Statistical analyses were performed by using 1-way ANOVA, Student's $t$ test (2-tailed test), or Chi-square test and significance was determined at $P$ less than 0.05 .

Study approval. Animal studies were approved by the Institutional Animal Care and Use Committee (IACUC) at Boston University Medical Center (protocol numbers 14388 and 15049). 


\section{Author contributions}

$\mathrm{XF}$ and WL conceived and designed the experiments. XF, HY, SK, KET, APH, HMR, RS, SC, ET, MS, and $\mathrm{MB}$ performed the experiments and acquired the data. XF, HY, SK, KET, APH, MB, SPB, and WL analyzed the data. JMH, AJC, DJS, SPB and WL provided reagents/materials/analysis tools. XF and WL wrote the manuscript.

\section{Acknowledgments}

We thank Jochen Reiser for providing podocyte cell lines, Janet Buhlmann for help with ROBO2-overexpressing cells, and Kelly Seidl for help with Operetta image analysis. This work is supported by NIH grant R01DK078226 (to WL), R01DK090029 (to DJS), a research grant from the Pfizer Centers for Therapeutic Innovation (to WL), a cooperative matching grant from the Massachusetts Life Sciences Center (to WL), and is also supported in part by Research Grant 1-FY12-426 from the March of Dimes Foundation (to WL).

Address correspondence to: Weining Lu, Associate Professor of Medicine, Renal Section, EBRC 538, Boston University Medical Center, 650 Albany Street, Boston, Massachusetts 02118, USA. Phone: 617.414.1770; E-mail: wlu@bu.edu

1. Tryggvason K, Patrakka J, Wartiovaara J. Hereditary proteinuria syndromes and mechanisms of proteinuria. $N$ Engl J Med. 2006;354(13):1387-1401.

2. Lennon R, Randles MJ, Humphries MJ. The importance of podocyte adhesion for a healthy glomerulus. Front Endocrinol (Lausanne). 2014;5:160.

3. Sachs N, Sonnenberg A. Cell-matrix adhesion of podocytes in physiology and disease. Nat Rev Nephrol. 2013;9(4):200-210.

4. Has C, et al. Integrin $\alpha 3$ mutations with kidney, lung, and skin disease. N Engl J Med. 2012;366(16):1508-1514.

5. Jones N, et al. Nck adaptor proteins link nephrin to the actin cytoskeleton of kidney podocytes. Nature. 2006;440(7085):818-823.

6. Verma R, Kovari I, Soofi A, Nihalani D, Patrie K, Holzman LB. Nephrin ectodomain engagement results in Src kinase activation, nephrin phosphorylation, Nck recruitment, and actin polymerization. J Clin Invest. 2006;116(5):1346-1359.

7. Fan X, et al. Inhibitory effects of Robo 2 on nephrin: a crosstalk between positive and negative signals regulating podocyte structure. Cell Rep. 2012;2(1):52-61.

8. Tessier-Lavigne M, Goodman CS. The molecular biology of axon guidance. Science. 1996;274(5290):1123-1133

9. Kidd T, et al. Roundabout controls axon crossing of the CNS midline and defines a novel subfamily of evolutionarily conserved guidance receptors. Cell. 1998;92(2):205-215.

10. Zallen JA, Yi BA, Bargmann CI. The conserved immunoglobulin superfamily member SAX-3/Robo directs multiple aspects of axon guidance in C. elegans. Cell. 1998;92(2):217-227.

11. Liu Z, Patel K, Schmidt H, Andrews W, Pini A, Sundaresan V. Extracellular Ig domains 1 and 2 of Robo are important for ligand (Slit) binding. Mol Cell Neurosci. 2004;26(2):232-240.

12. Morlot C, et al. Structural insights into the Slit-Robo complex. Proc Natl Acad Sci U S A. 2007;104(38):14923-14928.

13. Bashaw GJ, Kidd T, Murray D, Pawson T, Goodman CS. Repulsive axon guidance: Abelson and Enabled play opposing roles downstream of the roundabout receptor. Cell. 2000;101(7):703-715.

14. Wong K, et al. Signal transduction in neuronal migration: roles of GTPase activating proteins and the small GTPase Cdc42 in the Slit-Robo pathway. Cell. 2001;107(2):209-221.

15. Fan X, Labrador JP, Hing H, Bashaw GJ. Slit stimulation recruits Dock and Pak to the roundabout receptor and increases Rac activity to regulate axon repulsion at the CNS midline. Neuron. 2003;40(1):113-127.

16. Guan CB, Xu HT, Jin M, Yuan XB, Poo MM. Long-range $\mathrm{Ca}^{2+}$ signaling from growth cone to soma mediates reversal of neuronal migration induced by slit-2. Cell. 2007;129(2):385-395.

17. Rhee J, Buchan T, Zukerberg L, Lilien J, Balsamo J. Cables links Robo-bound Abl kinase to N-cadherin-bound beta-catenin to mediate Slit-induced modulation of adhesion and transcription. Nat Cell Biol. 2007;9(8):883-892.

18. Yang L, Bashaw GJ. Son of sevenless directly links the Robo receptor to rac activation to control axon repulsion at the midline. Neuron. 2006;52(4):595-607.

19. Grieshammer U, Le M, Plump AS, Wang F, Tessier-Lavigne M, Martin GR. SLIT2-mediated ROBO2 signaling restricts kidney induction to a single site. Dev Cell. 2004;6(5):709-717.

20. Lu W, et al. Disruption of ROBO2 is associated with urinary tract anomalies and confers risk of vesicoureteral reflux. Am $J$ Hum Genet. 2007;80(4):616-632.

21. Bertoli-Avella AM, et al. ROBO2 gene variants are associated with familial vesicoureteral reflux. J Am Soc Nephrol. 2008;19(4):825-831.

22. Hwang DY, et al. Mutations in 12 known dominant disease-causing genes clarify many congenital anomalies of the kidney and urinary tract. Kidney Int. 2014;85(6):1429-1433.

23. Hwang DY, et al. Mutations of the SLIT2-ROBO2 pathway genes SLIT2 and SRGAP1 confer risk for congenital anomalies of the kidney and urinary tract. Hum Genet. 2015;134(8):905-916.

24. Sellers JR. Myosins: a diverse superfamily. Biochim Biophys Acta. 2000;1496(1):3-22.

25. Bridgman PC. Growth cones contain myosin II bipolar filament arrays. Cell Motil Cytoskeleton. 2002;52(2):91-96.

26. Vicente-Manzanares M, Ma X, Adelstein RS, Horwitz AR. Non-muscle myosin II takes centre stage in cell adhesion and migration. Nat Rev Mol Cell Biol. 2009;10(11):778-790. 
27. Landsverk ML, Epstein HF. Genetic analysis of myosin II assembly and organization in model organisms. Cell Mol Life Sci. 2005;62(19-20):2270-2282.

28. Singh N, Nainani N, Arora P, Venuto RC. CKD in MYH9-related disorders. Am J Kidney Dis. 2009;54(4):732-740.

29. Johnstone DB, Ikizler O, Zhang J, Holzman LB. Background strain and the differential susceptibility of podocyte-specific deletion of Myh9 on murine models of experimental glomerulosclerosis and HIV nephropathy. PLoS One. 2013;8(7):e67839.

30. Johnstone DB, et al. Podocyte-specific deletion of Myh9 encoding nonmuscle myosin heavy chain 2A predisposes mice to glomerulopathy. Mol Cell Biol. 2011;31(10):2162-2170.

31. Recuenco MC, et al. Nonmuscle myosin II regulates the morphogenesis of metanephric mesenchyme-derived immature nephrons. J Am Soc Nephrol. 2015;26(5):1081-1091.

32. Lahoz A, Hall A. A tumor suppressor role for srGAP3 in mammary epithelial cells. Oncogene. 2013;32(40):4854-4860

33. Mundel $\mathrm{P}$, et al. Rearrangements of the cytoskeleton and cell contacts induce process formation during differentiation of conditionally immortalized mouse podocyte cell lines. Exp Cell Res. 1997;236(1):248-258.

34. Guan KL, Rao Y. Signalling mechanisms mediating neuronal responses to guidance cues. Nat Rev Neurosci. 2003;4(12):941-956

35. Grimsey NL, Moodley KS, Glass M, Graham ES. Sensitive and accurate quantification of human leukocyte migration using high-content Discovery-1 imaging system and ATPlite assay. J Biomol Screen. 2012;17(3):386-393.

36. Tamada M, Perez TD, Nelson WJ, Sheetz MP. Two distinct modes of myosin assembly and dynamics during epithelial wound closure. J Cell Biol. 2007;176(1):27-33.

37. Reidy KJ, Aggarwal PK, Jimenez JJ, Thomas DB, Veron D, Tufro A. Excess podocyte semaphorin-3A leads to glomerular disease involving plexinA1-nephrin interaction. Am J Pathol. 2013;183(4):1156-1168.

38. Delloye-Bourgeois C, et al. PlexinA1 is a new Slit receptor and mediates axon guidance function of Slit C-terminal fragments. Nat Neurosci. 2015;18(1):36-45.

39. Schordan S, et al. OPN deficiency results in severe glomerulosclerosis in uninephrectomized mice. Am J Physiol Renal Physiol. 2013;304(12):F1458-F1470.

40. Kirchhoff F, et al. Rapid development of severe end-organ damage in C57BL/6 mice by combining DOCA salt and angiotensin II. Kidney Int. 2008;73(5):643-650.

41. Kretzler M, Koeppen-Hagemann I, Kriz W. Podocyte damage is a critical step in the development of glomerulosclerosis in the uninephrectomised-desoxycorticosterone hypertensive rat. Virchows Arch. 1994;425(2):181-193.

42. Hiromura K, et al. Podocyte expression of the CDK-inhibitor p57 during development and disease. Kidney Int. 2001;60(6):2235-2246.

43. Pippin JW, et al. Cells of renin lineage are progenitors of podocytes and parietal epithelial cells in experimental glomerular disease. Am J Pathol. 2013;183(2):542-557.

44. Wang Y, Teng HL, Huang ZH. Repulsive migration of Schwann cells induced by Slit-2 through Ca ${ }^{2+}$-dependent RhoA-myosin signaling. Glia. 2013;61(5):710-723.

45. Barisoni L, Schnaper HW, Kopp JB. A proposed taxonomy for the podocytopathies: a reassessment of the primary nephrotic diseases. Clin J Am Soc Nephrol. 2007;2(3):529-542.

46. Wiggins RC. The spectrum of podocytopathies: a unifying view of glomerular diseases. Kidney Int. 2007;71(12):1205-1214.

47. D'Agati VD. Podocyte injury in focal segmental glomerulosclerosis: Lessons from animal models (a play in five acts). Kidney Int. 2008;73(4):399-406.

48. LeHir M, Kriz W. New insights into structural patterns encountered in glomerulosclerosis. Curr Opin Nephrol Hypertens. 2007;16(3):184-191

49. Lemley KV, et al. Podocytopenia and disease severity in IgA nephropathy. Kidney Int. 2002;61(4):1475-1485.

50. Toyoda M, Najafian B, Kim Y, Caramori ML, Mauer M. Podocyte detachment and reduced glomerular capillary endothelial fenestration in human type 1 diabetic nephropathy. Diabetes. 2007;56(8):2155-2160.

51. Pagtalunan ME, et al. Podocyte loss and progressive glomerular injury in type II diabetes. J Clin Invest. 1997;99(2):342-348.

52. Kriz W, Shirato I, Nagata M, LeHir M, Lemley KV. The podocyte's response to stress: the enigma of foot process effacement. Am J Physiol Renal Physiol. 2013;304(4):F333-F347.

53. Matsusaka T, et al. Podocyte injury damages other podocytes. J Am Soc Nephrol. 2011;22(7):1275-1285.

54. D'Agati V. Podocyte injury can be catching. J Am Soc Nephrol. 2011;22(7):1181-1183.

55. Topham PS, et al. Nephritogenic mAb 5-1-6 is directed at the extracellular domain of rat nephrin. J Clin Invest. 1999;104(11):1559-1566.

56. Ward ME, Rao Y. Investigations of neuronal migration in the central nervous system. Methods Mol Biol. 2005;294:137-156.

57. Eckly A, et al. Abnormal megakaryocyte morphology and proplatelet formation in mice with megakaryocyte-restricted MYH9 inactivation. Blood. 2009;113(14):3182-3189.

58. Ju Y, et al. Troponin T3 expression in skeletal and smooth muscle is required for growth and postnatal survival: characterization of Tnnt3(tm2a(KOMP)Wtsi) mice. Genesis. 2013;51(9):667-675.

59. Sachs N, et al. Blood pressure influences end-stage renal disease of Cd151 knockout mice. J Clin Invest. 2012;122(1):348-358

60. Sanden SK, Wiggins JE, Goyal M, Riggs LK, Wiggins RC. Evaluation of a thick and thin section method for estimation of podocyte number, glomerular volume, and glomerular volume per podocyte in rat kidney with Wilms' tumor-1 protein used as a podocyte nuclear marker. J Am Soc Nephrol. 2003;14(10):2484-2493.

61. Agrawal S, Guess AJ, Chanley MA, Smoyer WE. Albumin-induced podocyte injury and protection are associated with regulation of COX-2. Kidney Int. 2014;86(6):1150-1160.

62. Nieto-Pelegrin E, Kenny B, Martinez-Quiles N. Nck adaptors, besides promoting N-WASP mediated actin-nucleation activity at pedestals, influence the cellular levels of enteropathogenic Escherichia coli Tir effector. Cell Adh Migr. 2014;8(4):404-417. 